DOI 10. 18307/2021. 0403

(C) 2021 by Journal of Lake Sciences

\title{
中国湖泊生态系统突变时空差异”
}

董一凡 ${ }^{1,2}$, 郑文秀 ${ }^{1,2}$, 张晨雪 ${ }^{1,3}$, 徐 敏 ${ }^{1}$, 王 荣 ${ }^{1,4 * *}$, 吴兴华 ${ }^{5}$, 李 媛 $^{5}$

(1:中国科学院南京地理与湖泊研究所,南京 210008)

(2: 中国科学院大学, 北京 100049)

(3: 安徽师范大学, 芜湖 241002)

(4: 中国科学院抚仙湖高原深水湖泊研究站,玉溪 530422)

(5: 中国长江三峡集团有限公司, 北京 100038)

摘 要: 人类活动的加强导致湖泊生态系统发生“突变”, 造成生物多样性下降、藻类暴发、水质恶化等等环境和生态问 题. 中国许多湖泊已经发生 “突变”或面临着突变风险. 获悉湖泊生态系统发生突变的时空差异对于区域湖泊的保护, 预 防湖泊突变的发生以及制定合适的修复策略至关重要. 本研究收集了中国 55 个不同区域湖泊的古湖沼学数据, 探讨了湖 泊突变的区域特点. 研究确定了湖泊生态系统发生突变时间和区域差异, 并揭示了空间差异的原因. 结果表明, 中国湖泊 生态系统突变时间的区域分异特征为: 长江中下游湖泊最早出现突变; 东部和东北湖区湖泊突变时间明显早于西南、内 蒙古和新疆湖泊; 各湖区内, 城郊湖泊突变时间早于乡村湖泊. 人类活动强度是造成湖泊突变时空差异的主要驱动力. 研 究认为, 区域人类活动强度影响了湖泊生态系统的演化进程, 造成了中国湖泊生态系统突变的区域差异. 本研究从生态 系统突变的角度, 利用古湖沼学综述了中国湖泊生态系统在人类活动下的演化进程, 更深刻地认识了中国湖泊现状, 为 湖泊保护提供了有力的科学依据.

关键词: 中国湖泊;湖泊生态系统; 系统突变; 人类活动; 区域差异

\section{Temporal and spatial differences of lake ecosystem regime shift in China*}

\author{
Dong Yifan $^{1,2}$, Zheng Wenxiu ${ }^{1,2}$, Zhang Chenxue ${ }^{1,3}$, Xu Min ${ }^{1}$, Wang Rong ${ }^{1,4 * *}$, Wu Xinghua ${ }^{5} \& \operatorname{Li}^{\text {Yuan }}{ }^{5}$ \\ (1: Nanjing Institute of Geography and Limnology, Chinese Academy of Sciences, Nanjing 210008, P.R. China) \\ (2: University of Chinese Academy of Sciences, Beijing 100049, P.R.China) \\ (3: Anhui Normal University, Wuhu 241002, P.R.China) \\ (4: Fuxian Lake Research Station of Plateau-Deep Lake, Chinese Academy of Sciences, Yuxi 530422, P.R.China) \\ (5: China Three Gorges Corporation, Beijing 100038, P.R.China)
}

Abstract: Increased human activity has caused a regime shift in lake ecosystem, which causes environmental and ecological problems such as biodiversity decline, algae blooms and deterioration of water quality. Many Chinese lakes have undergone regime shift or are facing the risk of abrupt change. It is essential to know the temporal and spatial differences of regime shift for the protecting lake ecosystem, preventing regime shift and formulating appropriate restoration strategies. This study collected paleolimnology data of 55 lakes in different regions of China and discussed the regional characteristics of lake mutations. The study determined the temporal and spatial differences of regime shift in lake ecosystem and revealed the reasons for the spatial differences. Our study found that the regime shift time of Chinese lakes has the characteristics of regional differentiation: regime shift time of lakes in the middle and lower reaches of the Yangtze River is much earlier than that of the whole country. Regime shift happens earlier in the east and northeast lake areas than in the southwest, Inner Mongolia and Xinjiang lakes. In various lake region, regime shift happens earlier in the suburban lakes than in rural lakes. The intensity of human activities is the main driving force for the temporal and spatial

* 2020-09-30 收稿; 2020-12-11 收修改稿.

国家重点基础研发计划(2017YFA0605203)、中国科学院青年创新促进会项目 (2017364) 和中国长江三峡集团有 限公司项目 (201903144) 联合资助.

** 通信作者; E-mail: rwang@ niglas.ac.cn. 
differences of regime shift. Lakes with slower warming but stronger human activities are more likely to undergo regime shift. Our result suggests that the intensity of regional human activities has affected the evolution progress of lake ecosystems and caused regional differences of the regime shift in Chinese lakes. From the perspective of abrupt change in ecosystem, this study uses paleolimnology data to summarize the evolution of Chinese lake ecosystems under human activities, achieves a deeper understanding of the current situation of Chinese lakes, and provides a strong scientific basis for lake protection.

Keywords: Chinese lakes; lake ecosystems ; regime shift; human activities ; regional differences

在人类活动和气候变化驱动下湖泊生态系统可能发生突变 ${ }^{[1]}$, 造成对生态系统服务的巨大影响, 且难 以治理 ${ }^{[2]}$. 全球变化加速背景下, 由于资源的不合理利用和过度开发, 越来越多的湖泊营养上升、面临日益 严峻的突变风险 ${ }^{[3-4]}$. 第二次湖泊调查结果显示, 我国东部、东北和西南三大湖区约 $84.7 \%$ 的湖泊出现富营 养化, 其中长江中下游地区尤为严峻; 蒙新湖区、西南湖区湖泊营养梯度大, 贫营养湖泊和富营养湖泊共存; 青藏高原湖泊营养水平相对较低, 但区域增温正驱使该地区生态系统突变风险升高 ${ }^{[5]}$. 探究造成我国湖泊 生态系统发生突变的主导因素和区域分异规律, 对于预防湖泊生态系统发生突变,规避突变风险十分有利.

生态系统演化一直是湖泊科学的重要研究方向, 其中针对突变的研究从阈值判定 ${ }^{[6]}$ 、驱动力 ${ }^{[7]}$ 和早期 预警 ${ }^{[8]}$ 等方面展开, 主要研究方法包括数值模拟 ${ }^{[9]}$ 、实验模拟 ${ }^{[10]}$ 、湖泊调查等 ${ }^{[11]}$. 遥感调查发现, 气候变化 和人类活动对湖泊的影响程度存在区域差异 ${ }^{[12-13]}$. 具体表现为, 我国北部湖泊主要对气候变化有明显的响 应 ${ }^{[14]}$, 南部湖泊对农业面源污染和土地利用变化的响应更为明显 ${ }^{[15-16]}$. 其他研究表明, 湖泊物理属性如水 深、面积、蓄水量等也影响了我国湖泊的营养状态 ${ }^{[17]}$.

以上研究结果多是以揭示湖泊现状差异为研究目标, 缺少湖泊生态系统变化的长期研究, 从而难以揭 示湖泊生态系统突变. 在湖泊生态系统长期演化研究方面, 可分为长期监测 ${ }^{[18]}$ 和古湖沼学 ${ }^{[8]}$ 重建 2 种方法. 我国湖泊监测记录覆盖面窄、时间短, 研究对象局限于部分重点湖泊 ${ }^{[18]}$, 难以大规模比较不同湖泊的演化进 程. 采用古湖沼学手段, 可以重建百千万年来湖泊生态系统演化进程, 比较不同湖泊历史时期生态系统状态 的差异. 目前, 已通过古湖沼学手段进行研究的湖泊数量多、分布广 ${ }^{[19]}$, 可以弥补监测方法的不足, 进行湖 泊生态系统的区域分异研究.

古湖沼学使用沉积物代用指标揭示了湖泊生态系统突变, 明确了驱动力和突变机制. 研究指出, 农业活 动、工业化和城镇化等人类活动是造成湖泊生态系统突变的重要驱动力 ${ }^{[8,20]}$. 不同区域湖泊生态系统突变 模式、突变时间以及主要驱动力存在差异 ${ }^{[16]}$. 已有研究发现, 长江中下游和云南地区受到人类活动的影响, 湖泊生态系统演化过程中都存在突变; 长江中下游地区湖泊由于人类活动较强, 突变时间早于云南地区 ${ }^{[16]}$. 同时, 湖泊生态系统突变的驱动机制存在区域差异, 长江中下游湖泊都为浅水湖泊, 筑坝引起的水动力变化 以及沉水植物消失是湖泊生态系统发生突变的主要原因 ${ }^{[21]}$; 而云南高山湖泊以及深水湖泊生态系统的快速 变化也可能是由于外源氮沉降以及沉积物营养释放造成的 ${ }^{[8,22]}$.

前期的研究充分说明,在全球变化背景下,我国湖泊生态系统突变存在明显的区域差异. 但目前缺少全 国尺度上对湖泊生态系统突变点和驱动力时空差异的研究. 本研究通过整理我国湖泊的古湖沼学数据, 判 断湖泊状态突变的时间节点, 从全国湖泊生态系统演化突变点的角度分析湖泊生态系统突变的时空差异及 其产生的原因,为我国湖泊的管理与保护提供科学依据.

\section{1 研究方法与数据来源}

\section{1 数据选取}

本研究搜集了 73 个沉积物序列数据,包含东部湖区 21 个湖泊、东北湖区 6 个湖泊、内蒙古湖区 5 个湖 泊、新疆湖区 4 个湖泊和西南湖区 18 个湖泊 (湖泊分布和自然属性等见图 1 以及附表 I 和附图 I ${ }^{[20-21,23-71]}$. 若一个湖泊存在多个沉积序列, 本研究选取靠近湖心、分辨率较高的沉积记录代表该湖泊.

本研究重点选取能够指示湖泊富营养化的有效指标. 湖泊富营养化会提高湖泊初级生产力, 使沉积物 中有机质含量升高. 本研究选用总有机碳 ( TOC) 、烧失量 ( LOI) 、有机质含量 (OM) 指示湖泊生态系统初级 生产力的变化. 本研究排除了沉积物碳氮比较大的沉积序列 $(\mathrm{C}: \mathrm{N}$ 比 $>20)$.

研究以人类活动 (县域人均地区生产总值)、湖泊物理属性 (水深、面积、蓄水量) 与自然地理背景 (温 
度、降水、海拔) 作为自变量, 以湖泊生态系统突变的时间节点作为研究的因变量. 本研究使用《中国县域统 计年鉴 2018》所载的 2017 年度人均地区生产总值 (GDP) 统一衡量流域人类活动的强度 ${ }^{[72]}$. 若湖泊横跨多 个县域, 则以县域总常驻人口和 GDP 计算获得的人均 GDP 代替. 湖泊物理属性和海拔来源于相应文献和 《中国湖泊调查报告》 ${ }^{[73]}$. 研究使用的多年平均气温和降水数据, 根据湖泊点位通过 ArcGIS 10.2 从《中国 10 年平均水热条件变化公里网格数据集 $(1951-2010) 》$ 中提取 ${ }^{[74]}$.

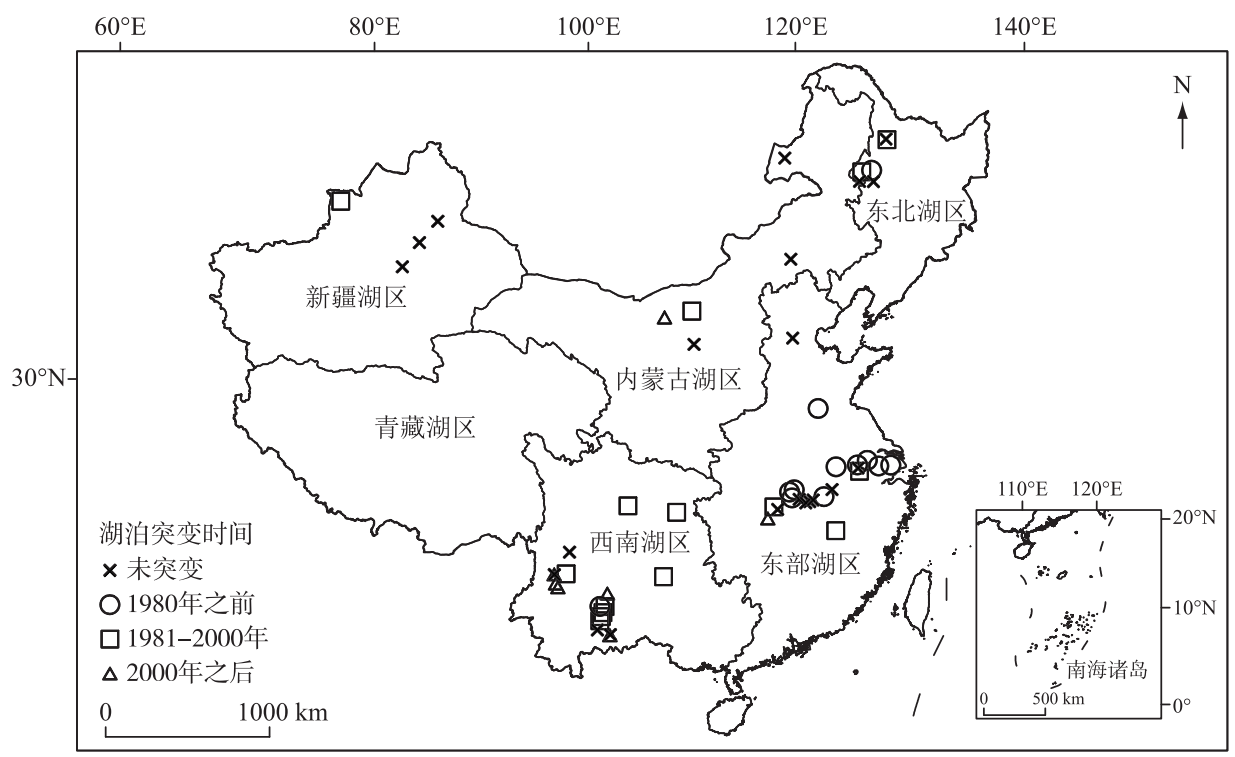

图 1 湖泊位置和湖泊生态系统突变时空分异

(东部和东北湖区湖泊突变集中于 $1980 \mathrm{~s}$ 之前,时间上明显早于西南、内蒙古和新疆湖泊.

该图基于自然资源部标准地图服务网站下载的审图号为 GS(2016)1600 号的标准地图制作,底图无修改)

Fig.1 Location and temporal-spatial differentiation of tipping point in lake ecosystem

\section{2 突变点检测}

本研究采用 $F$ 检验法、CUSUM 检验法分别检测时间序列的潜在突变点. 其中, $F$ 检验法采用 Chow 检 验,该方法假设序列中存在一点将序列区分为前后两组子序列, 使两个子序列各自的线性回归方程拟合优 度最高. 该方法通过计算 $F$ 统计量检测这一突变点:

$$
F=\frac{R S S-E S S}{E S S(n-2 k)}
$$

式中, $R S S$ 为时间序列上各点与总体线性回归方程的残差和, 计算每个点的 $F$ 统计量时均一致. 而每个点计 算的 ESS 为假设该点是突变点时, 前后两组数据相对各自线性回归方程的残差和. 而 $F$ 的峰值被认定为整 个序列发生剧烈变化的节点. $F$ 检验法在基于 $\mathrm{R}$ 平台的 Strucchange 结构检测包中进行 ${ }^{[75]}$, 设定的检测步长 为全序列的 $15 \% \sim 85 \%$.

CUSUM 检验法假定理想情况下, 距离均值最近的点最可能是突变点, 该方法通过计算各点与均值残差 的累计和来确定突变点 ${ }^{[76]}$ :

$$
\operatorname{CUSUM}_{x}=\sum_{i=1}^{n}\left(x_{n}-\bar{x}\right)
$$

如果序列总体上升, 则 $C U S U M_{x}$ 序列的极小值被认定是突变点; 如果序列总体上升, 则 CUSUM 序列的 极大值被认定是突变点. CUSUM 序列的峰度越大, 极值是突变点的可能性越高.

$F$ 检验法的优势在于识别出序列中的多个突变, 并鉴别突变点是否具有统计学意义, 但是可能将系统状 态的线性变化误认为是突变, 而 CUSUM 的峰度变化可以识别 $F$ 检验法的突变点是否发生于非线性变化中, 
与 $F$ 检验法互补. 因此本研究将综合两方法检验的结果, 以增加突变点识别的可靠性, 并结合案例研究中的 年代-深度对应关系,判定湖泊系统发生突变的时间.

\section{3 归因分析}

本研究按湖泊是否发生突变将案例分为两组,其中未突变组包括了指标没有显著变化或发生显著线性 响应的湖泊. 研究通过 $T$ 检验比较两组湖泊的自然属性和流域人类活动强度是否具有显著区别, 判断推动 生态系统发生突变而非渐变的关键因素. 对于发生突变的湖泊, 为了判断影响湖泊系统发生突变的主导因 素, 本研究将湖泊属性 (最大水深、面积、蓄水量)、湖泊自然地理背景 (多年平均水位、1950s 与 1970s 两时期 积温和平均降水差异值) 和人类活动强度作为自变量, 以湖泊突变的关键时间节点作为因变量进行多元回 归分析. 研究以多元线性回归中各自变量的回归系数 (Estimate) 绝对值作为评定各自变量对因变量影响相 对重要性的依据, 回归系数为通过最小二乘法确定的标准化回归系数. 研究以 $P$ 值作为检验各自变量对因 变量影响是否显著的依据.

\section{2 研究结果}

\section{1 湖泊突变的时空分异特征}

在被选取指标的总体变化趋势方面, 研究发现大部分湖泊的被选取指标与 $1950 \mathrm{~s}$ 相比, 在 21 世纪初均 有较大的上升或下降. 约 $52.73 \%$ 的湖泊, 被选取指标增长幅度超过 $50 \%$; 约 $16.36 \%$ 的湖泊, 上升幅度在 $25 \% \sim 50 \%$ 之间; 约 $14.55 \%$ 的湖泊，上升幅度在 5\% 25\% 之间; 约 10\% 的湖泊被选取指标含量下降超过 5\%, 其中 3.64\% 的湖泊被选取指标含量下降超过 $25 \%$. 仅 5\% 的湖泊,其变化不超过 $5 \%$. 总体上, 大多数湖泊沉 积物中 TOC 等指标在历史时期发生了明显变化.

在突变点检测方面, 本研究 55 个湖泊中有 34 个湖泊检测出突变点, 突变点检出比例为 $63 \%$. 其中, 发 生突变最早的湖泊为湖北涨渡湖, 突变时间为 1959 年前后; 最晚的湖泊为云南剑湖, 突变时间为 2009 年前 后. 根据案例数据来源文献论述, 突变点指示了湖泊生态系统状态转变的不同形式. 其中包括典型的由草型 湖变成藻型湖的草藻转换, 如武昌东湖 ${ }^{[63]} 、$ 滇池 ${ }^{[32]}$ 、程海 ${ }^{[27]}$ 、剑湖 ${ }^{[48]}$; 水生植被快速生长, 如淀山湖 ${ }^{[35]}$ 、梁 子湖 ${ }^{[52]}$ 、杞麓湖 ${ }^{[55]}$; 藻型湖泊的藻类暴发, 如星云湖 ${ }^{[68]}$; 以及藻类群落组成发生显著变化, 如巢湖 ${ }^{[25]}$ 、大理 西湖 ${ }^{[34]}$ 发生优势藻种的更替.

中国湖泊突变时间 (湖区划分依据参考文献[13-14]) 具有明显的空间分异特征 (图 1). 早期发生突变 的湖泊主要位于东部湖区和东北湖区, 即我国的长江中下游、华北和东北三大平原上. 其中, 东部湖区发生 突变最早的湖泊是涨渡湖, 1959 年即发生突变; 该湖区湖泊多于 1970s 发生突变, 例如淀山湖、太湖、长荡 湖、石臼湖和武昌东湖在 1974 年之前发生突变. 东部地区和东北地区仅东洞庭湖于 21 世纪发生突变. 西部 地区湖泊除滇池, 突变均发生于 1980s 后期到 21 世纪初. 西南湖区湖泊中, 发生突变最早的是滇池, 于 1970 年发生突变; 星云湖、杞麓湖等位于城市附近的湖泊多在 1990s 发生突变; 程海、清水海等距离城市较远的湖 泊, 检测出的突变点在 21 世纪初; 西南湖区中, 最晚发生突变的是剑湖, 于 2009 年发生突变. 内蒙古和新疆 两地共 10 处案例中有 3 处发生突变, 且时间均晚于 1990s. 同时, 研究结果显示城市湖泊多在 1970-1985 年 间发生突变, 而乡村湖泊发生突变的时间在 1990-2000 年间, 表现出明显的城乡差异 (图 2b). 综上所述, 我 国东部湖区尤其是长江中下游地区湖泊的突变最早, 东部、东北湖泊的突变明显早于西部湖泊 (图 2a), 在地 域分异基础上,城市湖泊的突变相对早于乡村湖泊(图 2b).

\section{2 突变主要的驱动力}

不同因素对湖泊是否发生突变影响 $T$ 检验分析结果显示, 温度差值通过了 $P \leqslant 0.05$ 的显著性检验, 发生 突变的湖泊平均气温下降了 $0.05^{\circ} \mathrm{C}$, 未发生突变的湖泊平均温度上升了 $0.35^{\circ} \mathrm{C}($ 表 1$)$. 人均 $\mathrm{GDP}$ 通过了 $P \leqslant$ 0.1 的显著性检验. 两组湖泊在其余属性上没有显著差异. 即温度未显著上升、但流域人类活动强度较高的 湖泊更容易发生突变.

湖泊面积、水深、蓄水量、海拔数据经对数化处理后, 与温度差值、降水量差值和人均地区生产总值一起 进行归一化处理. 归一化后的一元线性回归结果显示, 人类活动强度和水位分别通过了 $P \leqslant 0.01$ 的显著性 检验, 且回归系数绝对值均较大. 降水则通过了 $P \leqslant 0.05$ 的显著性检验, 且回归系数绝对值较大. 归一化后 

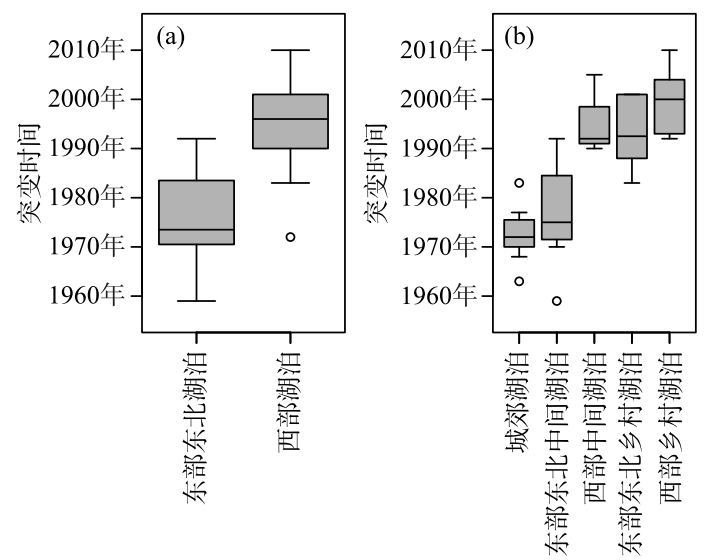

图 2 湖泊生态系统突变区域分异 (a) 和区域城乡分异 $(b)$

(东部湖泊突变总体上早于西部湖泊,在区域分异基础上,城郊湖泊突变早于乡村湖泊)

Fig.2 Regional differentiation (a) and urban-rural differentiation (b) of tipping point in lake ecosystem

的多元线性回归结果显示, 人类活动强度的回归系数绝对值最高且与突变时间显著相关 $(P \leqslant 0.05)$, 最大水 深通过了 $P \leqslant 0.1$ 的显著性检验并且回归系数绝对值也较大. 湖泊的面积、蓄水量、温度在一元和多元线性 回归中均未通过显著性检验, 与湖泊突变时间的关系不明确. 据一元线性回归结果和多元线性回归结果推 测, 我国湖泊突变主要驱动力是人类活动强度.

表 $1 T$ 检验和线性回归分析结果

Tab.1 $T$ test analysis result and linear regression analysis result

\begin{tabular}{|c|c|c|c|c|c|c|c|c|c|c|}
\hline \multirow[b]{2}{*}{ 自变量 } & \multicolumn{3}{|c|}{ 差异 $T$ 检验分析结果 } & \multicolumn{3}{|c|}{ 一元线性回归分析结果 } & \multicolumn{4}{|c|}{ 多元线性回归分析结果 } \\
\hline & $\begin{array}{l}\text { 未突变 } \\
\text { 组均值 }\end{array}$ & $\begin{array}{c}\text { 突变组 } \\
\text { 均值 }\end{array}$ & $P$ 值 & $\begin{array}{l}\text { 回归 } \\
\text { 系数 }\end{array}$ & $R^{2}$ & $P$ 值 & $\begin{array}{l}\text { 回归 } \\
\text { 系数 }\end{array}$ & 标准误 & $t$ 值 & $P$ 值 \\
\hline 人均 GDP/(万元) & 4.29 & 5.85 & 0.089 & -34.55 & 0.39 & $<0.01$ & 1.27 & 3.00 & 0.42 & 0.676 \\
\hline 面积 $/ \mathrm{km}^{2}$ & 250.75 & 392.38 & 0.460 & -1.96 & 0.09 & 0.091 & 9.39 & 5.09 & 1.85 & 0.079 \\
\hline 最大水深/m & 10.20 & 17.87 & 0.385 & 5.37 & 0.19 & 0.014 & -1.07 & 2.45 & -0.44 & 0.665 \\
\hline 蓄水量 $/\left(\times 10^{8} \mathrm{~m}^{3}\right)$ & 7.37 & 18.68 & 0.991 & -1.10 & 0.06 & 0.210 & 1.72 & 3.29 & 0.52 & 0.607 \\
\hline 多年平均水位/m & 620.17 & 794.21 & 0.462 & 3.87 & 0.44 & $<0.01$ & 5.38 & 8.13 & 0.66 & 0.516 \\
\hline 温度差值 $/{ }^{\circ} \mathrm{C}$ & 0.35 & -0.05 & 0.018 & 3.60 & 0.20 & 0.655 & 1.55 & 6.96 & 0.22 & 0.826 \\
\hline 降水量差值/mm & 1.19 & 0.34 & 0.976 & 13.59 & 0.13 & 0.036 & -20.51 & 9.17 & -2.24 & 0.036 \\
\hline
\end{tabular}

图 3 进一步展示了人类活动强度与湖泊突变时间回归关系. 图 3 显示突变时间与人均地区生产总值存 在明确的统计学意义. 不同地区湖泊的突变时间也展现出差别: 东部湖泊的突变时间较早, 西南、内蒙古和 新疆湖泊突变时间较晚 (图 3a) ; 城市湖泊突变相对较早, 而乡村湖泊突变相对较晚 (图 3b) ; 突变早晚差别 与这些地区的人类活动的强弱相关.

\section{3 讨论}

本研究通过古湖沼学数据确定了我国不同湖泊突变的时间节点, 并对湖泊突变的主要驱动力进行了分 析. 国际相关研究认为湖泊物理属性和气候等是自然条件影响湖泊突变进程的主要因素 ${ }^{[7]}$. 本研究的多元 线性回归结果显示, 湖泊物理属性仅水深展现出深水湖泊突变时间较晚的特征, 这可能是由于东部地区多 浅水湖, 湖泊深度与流域人类活动强度存在共线性. 长江中下游湖泊突变较早且当地经济发展水平相对较 高, 海拔在一元回归分析中的显著性和影响可能来自这一地区的海拔较低. 已有研究认为气温升高会造成 

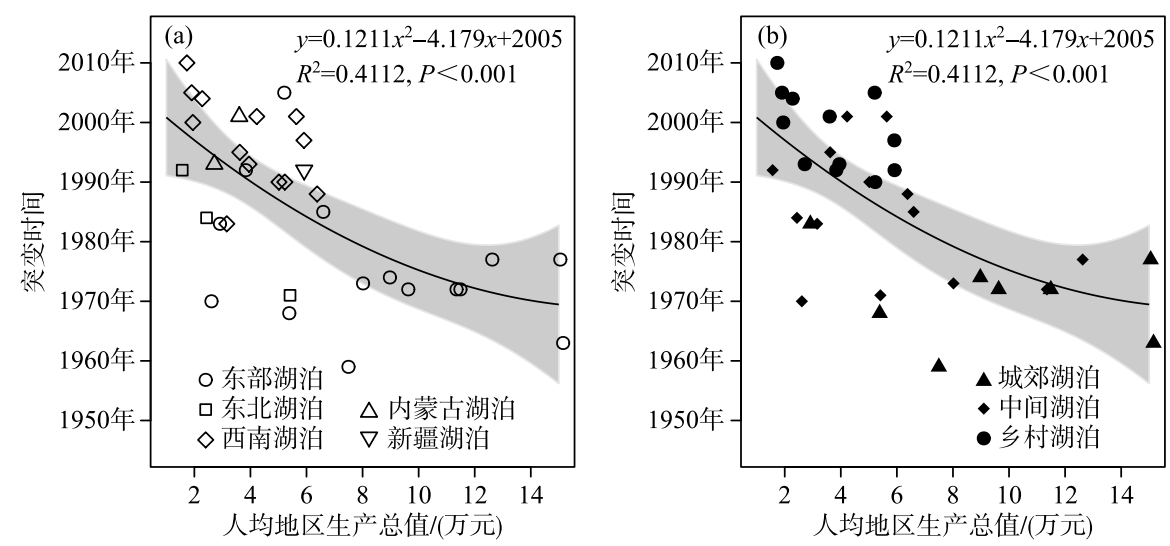

图 3 流域人类活动强度与湖泊突变时间回归关系

(湖泊突变时间与人均地区生产总值存在统计学意义,人均地区生产总值越高, 突变越早)

Fig.3 Regression relationship between the intensity of human activities and the abrupt change time of lakes

生态系统突变 ${ }^{[7]}$,但本研究发现未突变的湖泊升温趋势更明显. 降水在一元线性回归中较为显著,但在多元 回归中重要性并不大, 说明降水的变化并不是突变时间先后的主导因素. 本研究认为人类活动对湖泊突变 进程的影响远远超过了湖泊的物理属性和气候特征, 中国湖泊生态系统突变的时空格局主要由人类活动的 区域差异造成,具体体现在经济发展水平差异和土地利用差异两方面.

经济发展方面,农业活动产生的流域面源营养排放是湖泊营养的主要外源,农业化肥通过淋溶作用汇 人湖泊,显著提高了湖泊的营养水平 ${ }^{[77]} .1980 \mathrm{~s}$ 初, 我国已形成了与湖泊突变时间格局相似的化肥施用区域 不平衡格局, 即东部高于西部; 近郊乡村高于远郊及山区 ${ }^{[78]}$. 根据《中国农业年鉴 1981》, 1980 年, 长江中下 游地区沪苏皖鄂四省市农业施肥量按纯折量达到 $13.65 \mathrm{~g} / \mathrm{m}^{2}$, 高于全国同期水平 $\left(10.8 \mathrm{~g} / \mathrm{m}^{2}\right)$, 远高于本研 究中涉及较多的云南 $\left(7.95 \mathrm{~g} / \mathrm{m}^{2}\right)$ 和黑龙江 $\left(4.8 \mathrm{~g} / \mathrm{m}^{2}\right)^{[79]}$. 本研究结果显示, $1980 \mathrm{~s}$ 前发生突变的湖泊主要 集中于这一地区 (图 3). 农业发展还会带动农村如畜禽养殖、水产养殖在内的其他产业发展. 人口密度越 大、畜禽养殖密度就越高, 而流域内的畜禽粪肥是湖泊营养的重要来源. 畜禽养殖格局存在城乡差异, 为满 足城市居民生活需要, 大型机械化养殖场多修建在城乡结合部, 使城郊的养殖密度高于乡村、更容易造成营 养元素排人水系 ${ }^{\left[{ }^{[0]}\right.}$. 在畜禽养殖格局方面, 东部无论是地均还是人均养殖强度均显著高于西部, 但 $1990 \mathrm{~s}$ 以 来该差距大大收窄 ${ }^{[81]}$. 畜禽养殖格局重心的总体西移的时间与西部地区湖泊开始突变的时间接近, 说明西 部地区湖泊人湖营养可能和畜牧业发展有关. 例如, 流域奶牛养殖数量的增加, 对洱海 ${ }^{[8]}$ 的营养富集有重要 影响. 淡水渔业养殖通过大量投放渔肥提高营养富集、通过提高鱼类产量降低沉水植被生物量 ${ }^{[82]}$. 已经研 究还发现优势种更替可能加速湖泊突变 ${ }^{[83]}$, 而引入外来鱼种可能使本地鱼类种群数量急剧减少, 造成优势 种更替. $1980 \mathrm{~s}$ 后, 云南程海、抚仙湖、星云湖等湖泊引人 “四大家鱼” 和太湖银鱼养殖, 这些湖泊的物种更替 时间与发生突变的时间吻合 ${ }^{[27,39,68]}$.

工业是人类活动强度影响湖泊突变进程的另一体现. 工业发展会产生携带有机营养盐的生产污水, 还 会促进城镇形成、提高人口密度、产生更多的生活污水, 二者均会加速湖泊营养富集. 1950s 初, 我国已形成 沿海沿江地区工业产值和就业人口均远高于内陆地区的工业化和城市化格局 ${ }^{[84]}$. 这一格局对长江中下游 湖泊突变早于其他地区, 对城区湖泊突变早于乡村湖泊具有影响. 如昆明市位于滇池岸, 市内主要径流盘龙 江直接流人滇池, 滇池是西南地区唯一突变早于 1985 年的湖泊,可能与昆明较强的工商业发展有关.

人类活动强度增强的另一大体现是土地利用变化,而土地利用变化会通过旺荒和城市化加速湖泊营养 富集. 流域旺荒加剧流域土壤侵蚀使得土壤中的营养元素被搬运人湖泊; 同时旺荒会截留人湖水量,减缓湖 泊水体更新. 东北地区土壤以有机质高的黑钙土为主, 旺荒造成大量土壤营养元素被搬运人湖泊、加速营养 富集 ${ }^{[85]}$. 黑龙江克钦泡、鸿雁泡、五大连池自 19 世纪开始圼荒, 流域侵蚀不断增强、人湖营养不断提高, 在 
20 世纪相继发生突变.

诸多研究表明, 人类活动通过建闸筑坝和围剭湖面, 改变了湖泊的物理属性, 会严重影响湖泊的突变时 间 ${ }^{[86]}$. 围垦活动破坏湖岸湿地、降低了湖泊对外源营养物的抵抗力, 加强流域农业强度活动、提高人湖营养 水平, 缩小湖泊面积、提高了湖泊水体营养浓度. 已有研究发现, 过去 50 年人类活动对我国东部和西南地区 湖泊面积变化重大影响, 其中围垦的影响显著 ${ }^{[13]}$. 湖北洪湖、涨渡湖等均受到严重围垦. 建闸筑坝则会提高 湖泊水位, 使藻类代替草类成为湖泊初级生产力承担者,加速 “草一藻” 转换进程. 如涨渡湖在建闸第二年即 发生重大突变 ${ }^{[22]}$. 长江中下游湖泊仅洞庭湖、鄱阳湖和石臼湖未建坝筑闸 ${ }^{[5]}$, 对长江流域湖泊突变进程明 显早于全国可能有影响. 本研究虽然没有发现湖泊物理属性与突变时间存在显著关系, 但是通过上述人类 活动方式改变了物理属性, 显然改变了湖泊突变的进展, 其中进一步的关系需要更多的研究揭示.

本研究认为, 流域人类活动是塑造我国湖泊突变时空差异的主要驱动力. 我国沿海和沿江地区人口稠 密、工商业发展早、高密度施肥早、渔业养殖量大, 而东北地区近代农业开发强度大、土壤有机质背景高, 使 这一地区的湖泊营养富集更早达到突变阈值. 随着社会经济发展, 西部地区农业畜牧业的发展使得本地区 湖泊的营养水平不断提高, 湖泊系统发生突变. 特定的人类活动, 如云南引人外来鱼种、长江中下游湖泊严 重的围垦活动均加速了当地湖泊生态系统演化. 人口密度提高和生产生活需要使城市排放营养浓度较高的 污水,加速了城郊湖泊的营养富集过程.

前人关于湖泊系统响应气候变化和人类活动的区域差异研究, 主要集中在水质和水量变化两方面. 水 质方面, 营养富集在各湖区均造成不同程度的水质问题, 总体上以东部更严重 ${ }^{[5,15]}$; 水量方面, 生活生产用水 增加和降水减少造成了不同地区湖泊人湖水量下降, 使北部湖泊的面积萎缩 ${ }^{[15,87-89]}$. 气候变化逐渐加强的 背景下, 人类活动影响的区域格局不仅塑造了前人研究得出的水质、水量变化格局, 还深刻影响了湖泊生态 系统的突变时空差异格局. 前人研究结合本研究对湖泊突变时间节点的判断, 可以得出我国湖泊对气候变 化和人类活动响应的空间分异格局: 东部湖区受人类活动影响最早、最明显, 湖泊面积明显减少、富营养化 程度提高, 其中长江中下游湖泊还存在严重江河阻断, 使湖泊生态系统突变较早; 东北湖区湖泊受气候变化 和人类活动影响较为剧烈, 水质下降、水量减少, 湖泊生态系统发生突变较早; $1980 \mathrm{~s}$ 以来, 随着全球气候变 化加速、区域经济发展加快, 西南湖区湖泊营养富集加速, 水质、水量和生态问题日益凸显, 但相对东部地区 较轻, 生态系统突变相对较晚; 内蒙古和新疆湖区湖泊在气候变化影响下, 部分湖泊水量明显减少, 生态系 统突变较少、较晚.

本研究从系统突变的角度揭示了人类活动驱动下湖泊生态系统演化的格局, 是认识中国湖泊现状、保 护湖泊资源等重大课题的有力依据. 除格局研究, 本研究还在湖泊生态系统演化的驱动力方面具有参考价 值. 虽然本研究认为海拔与人类活动主导的突变进程可能仅存在协相关, 但目前研究均认为高山高原湖泊 对气候变化的响应更敏感, 考虑到湖泊系统受气候变化的影响越来越大, 未来研究和湖泊管理应更加关注 这一背景对高山高原湖泊的影响. 进一步研究还应关注生态系统对外力胁迫响应方式的选择倾向.

\section{4 结论}

中国湖泊突变时间的空间差异主要是长江中下游湖泊明显最早, 东部和东北湖泊明显早于西部湖泊; 在区域分异基础上, 城郊湖泊发生突变相对早于乡村湖泊. 研究认为, 空间分异的主要驱动力来自于人类活 动, 人均 GDP 的差异明显影响了湖泊的突变时间. 在气候变化方面, 降水下降可能加速了湖泊突变, 气温升 高可能使得湖泊生态系统发生渐变而非突变. 全球变化大背景下, 人类活动是造成我国湖泊系统从水量、水 质到生态系统整体发生剧烈变化的主要原因, 人类活动的区域差异不仅塑造了中国湖泊的水质水量变化格 局,还深刻影响了中国湖泊突变时间的空间差异.

\section{5 附录}

附表 I 和附图 I 见电子版( DOI: 10.18307/2021.0403).

\section{6 参考文献}

[ 1 ] Scheffer M, Hosper SH, Meijer ML et al. Alternative equilibria in shallow lakes. Trends in Ecology \& Evolution, 1993, 8 
( 8 ) : 275-279. DOI: 10.1016/0169-5347( 93 ) 90254-m.

[ 2 ] Scheffer M, Carpenter S, Foley JA et al. Catastrophic shifts in ecosystems. Nature, 2001, 413(6856) : 591-596. DOI: $10.1038 / 35098000$.

[ 3 ] Liu J, Yang W. Water sustainability for China and beyond. Science, 2012, 337 (6095) : 649-650. DOI: 10.1126/ science.1219471.

[ 4 ] Ho JC, Michalak AM, Pahlevan N. Widespread global increase in intense lake phytoplankton blooms since the 1980s. Nature, 2019, 574(7780) : 667-670. DOI: 10.1038/s41586-019-1648-7.

[ 5 ] Yang GS, Ma RH, Zhang L et al. Lake status, major problems and protection strategy in China. J Lake Sci, 2010, 22 (6) : 799-810. DOI : 10.18307/2010.0601. [杨桂山, 马荣华, 张路等. 中国湖泊现状及面临的重大问题与保护策 略. 湖泊科学, 2010, 22(6): 799-810.]

[ 6 ] Scheffer M, Carpenter SR. Catastrophic regime shifts in ecosystems: Linking theory to observation. Trends in Ecology \& Evolution, 2003, 18(12) : 648-656. DOI: 10.1016/j.tree.2003.09.002.

[ 7 ] Scheffer M, Nes EH. Shallow lakes theory revisited: Various alternative regimes driven by climate, nutrients, depth and lake size. Hydrobiologia , 2007, 584(1) : 455-466. DOI: 10.1007/s10750-007-0616-7.

[ 8 ] Wang R, Dearing JA, Langdon PG et al. Flickering gives early warning signals of a critical transition to a eutrophic lake state. Nature, 2012, 492(7429) : 419-422. DOI: 10.1038/nature11655.

[ 9 ] Mooij WM, Janse JH, Senerpont Domis LN et al. Predicting the effect of climate change on temperate shallow lakes with the ecosystem model PCLake. Hydrobiologia, 2007, 584(1) : 443-454. DOI: 10.1007/s10750-007-0600-2.

[10] Ding L, Li W, Shi B et al. Effects of water transparency on Potamogeton crispus growth and its dynamic model. Acta Ecologica Sinica, 2009, 29(1) : 92-96. [丁玲, 李巍, 施蓓等. 透明度胁迫对蕰草 (Potamogeton crispus) 生长的定量影 响及其生长动力学模型. 生态学报, 2009, 29(1): 92-96.]

[11] Su HJ, Wu Y, Xia WL et al. Stoichiometric mechanisms of regime shifts in freshwater ecosystem. Water Research, 2019, 149: 302-310. DOI: 10.1016/j.watres.2018.11.024.

[12] Tao SL, Fang JY, Ma SH et al. Changes in China's lakes: Climate and human impacts. National Science Review, 2020, 7 (1) : 132-140. DOI: $10.1093 / \mathrm{nsr} / \mathrm{nwz} 103$.

[13] Zhang GQ, Yao TD, Chen WF et al. Regional differences of lake evolution across China during 1960s-2015 and its natural and anthropogenic causes. Remote Sensing of Environment, 2019, 221: 386-404. DOI: 10.1016/j.rse.2018.11.038.

[14] Ma RH, Duan HT, Hu CM et al. A half-century of changes in China's lakes: Global warming or human influence? Geophysical Research Letters, 2010, 37(24) : L24106. DOI: 10.1029/2010GL045514.

[15] Fang JY, Rao S, Zhao SQ. Human-induced long-term changes in the lakes of the Jianghan Plain, Central Yangtze. Frontiers in Ecology and the Environment, 2005, 3(4) : 186-192. DOI: 10.1890/1540-9295(2005) 003 [0186: HLCITL]2.0. $\mathrm{CO} ; 2$.

[16] Zhang K, Dong XH, Yang XD et al. Ecological shift and resilience in China's lake systems during the last two centuries. Global and Planetary Change, 2018, 165: 147-159. DOI: 10.1016/j.gloplacha.2018.03.013.

[17] Ding JT, Cao JL, Xu QG et al. Spatial heterogeneity of lake eutrophication caused by physiogeographic conditions: An analysis of 143 lakes in China. Journal of Environmental Sciences, 2015, 30: 140-147. DOI: 10.1016/j.jes.2014.07.029.

[18] Chen XH, Qian XY, Li XP et al. Long-term trend of eutrophication state of Lake Erhai in 1988-2013 and analyses of its socio-economic drivers. J Lake Sci, 2018, 30(1) : 70-78. DOI: 10.18307/2018.0107. [陈小华, 钱晓雍, 李小平等. 洱 海富营养化时间演变特征 (1988-2013 年) 及社会经济驱动分析. 湖泊科学, 2018, 30(1) : 70-78.]

[19] Shen J, Zhang K, Liu ZW. Paleolimnological evidence of environmental change in Chinese lakes over the past two centuries. Inland Waters, 2020, 10(1) : 1-10. DOI: 10.1080/20442041.2019.1657348.

[20] Yang Y, Yin XN, Yang ZF et al. Detection of regime shifts in a shallow lake ecosystem based on multi-proxy paleolimnological indicators. Ecological Indicators, 2018, 92: 312-321. DOI: 10.1016/j.ecolind.2017.05.059.

[21] Xu M, Wang R, Dong XH et al. A palaeolimnological perspective to understand regime-shift dynamics in two Yangtze-basin lakes. Biology Letters, 2019, 15(11) : 20190447. DOI: 10.1098/rsbl.2019.0447.

[22] Hu ZJ, Anderson NJ, Yang XD et al. Catchment-mediated atmospheric nitrogen deposition drives ecological change in two alpine lakes in SE Tibet. Global Change Biology, 2014, 20(5) : 1614-1628. DOI: 10.1111/gcb.12435.

[23] Ding ZY, Ma L, Jilili A et al. Elemental geochemical records and its eco-environment significance of sediment core in 
ebinur lake, Xinjiang, China. Journal of Desert Research, 2018, 38(1) : 101-110. [丁之勇, 马龙, 吉力力·阿不都外 力等. 新疆艾比湖湖泊沉积物元素地球化学记录及其生态环境意义. 中国沙漠, 2018, 38(1): 101-110.]

[24] Zhang EL, Zheng BY, Cao YM et al. The effects of environmental changes on chironomid fauna during the last century in Bosten Lake, Xinjiang, NW China. Fundamental and Applied Limnology, 2012, 180(4) : 299-307. DOI : 10.1127/18639135/2012/0324.

[25] Chen X, Yang XD, Dong XH et al. Effects of environmental changes on the succession of diatom assemblage during the last 50 years in Lake Chaohu. J Lake Sci, 2011, 23(5) : 665-672. DOI: 10.18307/2011.0501. [陈旭, 羊向东, 董旭辉 等. 近 50 年来环境变化对巢湖硅藻组合演替的影响. 湖泊科学, 2011, 23(5): 665-672.]

[26] Zhang YD, Su YL, Liu ZW et al. Sedimentary lipid biomarker record of human-induced environmental change during the past century in Lake Changdang, Lake Taihu basin, Eastern China. Science of the Total Environment, 2018, 613/614: 907-918. DOI: 10.1016/j.scitotenv.2017.09.185.

[27] Zan FY, Huo SL, Xi BD et al. A 60-year sedimentary record of natural and anthropogenic impacts on Lake Chenghai, China. Journal of Environmental Sciences, 2012, 24(4) : 602-609. DOI: 10.1016/S1001-0742(11)60784-5.

[28 ] Wang JZ, Wu JL, Zeng HA. Sediment record of abrupt environmental changes in Lake Chenpu, upper reaches of Yellow River Basin, North China. Environmental Earth Sciences, 2015, 73 (10): 6355-6363. DOI: 10. 1007/s12665-0143857-6.

[29] Tao JS. The patterns of diatom community responses to pollution and hydrological regulation in low-latitude plateau lakes of southwest China[Dissertation]. Kunming: Yunnan Normal University, 2017. [ 陶建霜. 低纬高原湖泊污染与水文调控 的硅藻群落响应模式识别 [学位论文]. 昆明:云南师范大学, 2017.]

[30] Anjum R, Tang Q, Collins AL et al. Sedimentary chronology reinterpreted from Changshou Lake of the Three Gorges Reservoir Area reveals natural and anthropogenic controls on sediment production. Environmental Science and Pollution Research, 2018, 25(18) : 17620-17633. DOI: 10.1007/s11356-018-1916-3.

[31] Wu JL, Ma L. Characteristics of the climate and environment in arid regions over the past 150 years recorded by the core sediments of chaiwopu lake, Xinjiang, China. Quaternary Sciences, 2010, 30(6) : 1137-1144. [ 吴敬禄, 马龙. 新疆干 旱区柴窝堡湖沉积记录的 150 年来气候环境特征. 第四纪研究, 2010, 30(6) : 1137-1144.]

[32] Yu LY, Han XX, Huang XH et al. Distribution characteristics of n-alkanes in sediment core and implication of environment in different lakes of Dianchi. Environmental Science, 2016, 37(12) : 4605-4614. DOI: 10.13227/j.hjkx.201605151. [余丽燕, 韩秀秀, 黄晓虎等. 滇池不同湖区沉积物正构烷烃的分布特征及其环境意义. 环境科学, 2016, 37 (12) : 4605-4614.]

[33] Zhen ZL, Zhang S, Li WB. Environment variation in north margin of east Asian summer monsoon record in Dali lake over past 168 years. Water Resources, 2019, 46(2) : 172-181. DOI: 10.1134/S0097807819020180.

[34] Zhang CX, Xu M, Dong YF et al. Sedimentary diatom records reveal the succession of ecosystem in lake Xihu, Dali over the past 50 years. Environmental Science, 2020, 41(10): 4572-4580. DOI: 10.13227/j.hjkx.202003293. [张晨雪, 徐 敏, 董一凡等. 硅藻群落指示的近 50 年来大理西湖湖泊生态系统演变规律. 环境科学, 2020, 41 (10): 4572-4580. ]

[35] Li XP, Chen XH, Dong XH et al. Nutrient dynamics over the past 100 years and its restoration baseline in Dianshan lake. Environmental Science, 2012, 33(10) : 3301-3307. DOI: 10.13227/j.hjkx.2012.10.019. [李小平, 陈小华, 董旭辉等. 淀山湖百年营养演化历史及营养物基准的建立. 环境科学, 2012, 33(10): 3301-3307.]

[36] Tao JS, Chen GJ, Chen XL et al. Long-term pattern of diatom community structure changes in response to multiple environmental stressors at Datun Lake, Southeast Yunnan, China. Chinese Journal of Applied Ecology, 2015, 26( 8) : 25252533. DOI: 10.13287/j.1001-9332.20150610.010. [ 陶建霜, 陈光杰, 陈小林等. 多重环境压力下大屯海硅藻群落结 构的长期变化. 应用生态学报, 2015, 26(8) : 2525-2533.]

[37] Chen X, McGowan S, Zeng LH et al. Changes in carbon and nitrogen cycling in a floodplain lake over recent decades linked to littoral expansion, declining riverine influx, and eutrophication. Hydrological Processes, 2017, 31 (17) : 31103121. DOI: $10.1002 /$ hyp.11254.

[38] Liu GM, Liu ZW, Gu BH et al. How important are trophic state, macrophyte and fish population effects on cladoceran community? A study in Lake Erhai. Hydrobiologia, 2014, 736(1) : 189-204. DOI: 10.1007/s10750-014-1906-5.

[39] Liu EF, Zhang EL, Li K et al. Historical reconstruction of atmospheric lead pollution in central Yunnan Province, south- 
west China: An analysis based on lacustrine sedimentary records. Environmental Science and Pollution Research, 2013,20 (12) : 8739-8750. DOI: 10.1007/s11356-013-1861-0.

[40] Yao SC, Xue B, Wang XL. Environment changes influenced by human activities at Lake Gucheng. J Lake Sci, 2008, 20 (1) : 88-92. DOI : 10.18307/2008.0113. [姚书春, 薛滨, 王小林. 人类活动影响下的固城湖环境变迁. 湖泊科学, $2008,20(1): 88-92$.

[41] Yao SC, Xue B, Xia WL et al. Lead pollution recorded in sediments of three lakes located at the middle and lower Yangtze River basin, China. Quaternary International, 2009, 208(1/2) : 145-150. DOI: 10.1016/j.quaint.2008.10.013.

[42] Luo Y, Lu MH, Wang HY et al. Recent soil erosion in the Hongfeng catchment on the Guizhou Plateau, SW China revealed by analysis of reservoir sediments and soil loss modeling. Journal of Paleolimnology, 2019, 61(1) : 17-35. DOI: 10.1007/s10933-018-0042-z.

[43] Yao SC, Xue B. Sedimentary geochemical record of human-induced environmental changes in Huanggaihu Lake in the middle reach of the Yangtze River, China. Journal of Limnology, 2015, 74(1) : 31-39.. DOI: 10.4081/jlimnol.2014.957.

[44] Li S, Chen S, Zhang JW. Environmental changes recorded by lake sediments from Hongjiannao lake during recent fifty years. Journal of Anhui Agricultural Sciences, 2010, 38(5): 2534-2537, 2576. [李双, 陈硕, 张家武. 红碱淖湖泊沉 积记录的近五十年来的环境变化. 安徽农业科学, 2010, 38(5) : 2534-2537, 2576.]

[45] Gao HB, Li CY, Sun B. The impact of changed river discharge on water quality deterioration in a prairie lake revealed by the sedimentary evidence. Water Supply, 2018, 18(1) : 299-305. DOI: 10.2166/ws.2017.120.

[46] Wang JY, Chen GJ, Kang WG et al. Impoundment intensity determines temporal patterns of hydrological fluctuation, carbon cycling and algal succession in a dammed lake of Southwest China. Water Research, 2019, 148: 162-175. DOI: 10. 1016/j.watres.2018.10.032.

[47] MacKenzie L, Bao KS, Mao LM et al. Anthropogenic and climate-driven environmental change in the Songnen Plain of northeastern China over the past 200-years. Palaeogeography, Palaeoclimatology, Palaeoecology, 2018, 511: 208-217. DOI: $10.1016 /$ j.palaeo.2018.08.005.

[48] Liu S, Chen GJ, Wang JY et al. Diatom community response to long-term enviromental changes and ecological evaluation in Jianhu lake, northwest Yunnan. Quaternary Sciences, 2018, 38(4) : 939-952. [刘术, 陈光杰, 王教元等. 剑湖硅藻 群落对环境变化的长期响应模式与生态评价. 第四纪研究, 2018, 38(4) : 939-952.]

[49] Kong RR, Zhang ML, Yang H. Study on the characteristics of nutrient distribution of surface sediments under the influence of human activities in Honghu lake, Jiangxi Province. Advanced Materials Research, 2013, 864/865/866/867: 897-902. DOI: $10.4028 /$ www. scientific.net/amr.864-867.897.

[50] Bing HJ, Wu YH, Zhang YJ et al. Possible factors controlling the distribution of phosphorus in the sediment of Longgan Lake, middle reach of Yangtze River, China. Environmental Earth Sciences, 2014, 71(10) : 4553-4564. DOI: 10.1007/ s12665-013-2848-3.

[51] Zhang EL, Tang HQ, Cao YM et al. The effects of soil erosion on chironomid assemblages in Lugu Lake over the past 120 years. International Review of Hydrobiology, 2013, 98(3) : 165-172. DOI: 10.1002/iroh.201301468.

[52] Zhang QH, Dong XH, Yang XD et al. Hydrologic and anthropogenic influences on aquatic macrophyte development in a large, shallow lake in China. Freshwater Biology, 2019, 64(4) : 799-812. DOI: 10.1111/fwb.13263.

[53] Ding Zhaoyun. Study of dfifference between north and south of recent sedimentation of Nansi lake under human activities [Dissertation]. Nanjing: Nanjing Normal University, 2017. [丁兆运. 人类活动影响下南四湖现代沉积的南北差异研 究 [学位论文]. 南京:南京师范大学, 2017.]

[54] Tao YQ, Xue B, Yao SC et al. Triolein embedded cellulose acetate membrane as a tool to evaluate sequestration of PAHs in lake sediment core at large temporal scale. Environmental Science \& Technology, 2012, 46(7) : 3851-3858. DOI: 10. $1021 /$ es203102b.

[55] Qian FM, Zhang K, Chen GJ et al. Sediment-inferred recent pattern and drivers of environmental and ecological changes at Lake Qilu, Yunnan Province. J Lake Sci, 2018, 30(4) : 1109-1122. DOI: 10.18307/2018.0423. [ 钱福明, 张恺, 陈光 杰等. 云南杞麓湖沉积物记录的近现代生态环境变化及影响因子识别. 湖泊科学, 2018, 30(4): 1109-1122.]

[56] Jia BY, Fu WL, Yu J et al. Relationship among sediment characteristics, eutrophication process and human activities in the Sancha Lake, Sichuan, Southwestern China. China Environmental Science, 2013, 33(9): 1638-1644. [贾滨洋, 付 文丽, 于静等. 三岔湖沉积物揭示的湖泊营养化进程与人类活动的关系. 中国环境科学, 2013, 33(9): 
1638-1644.]

[57] Yao SC, Xue B. Recent environmental evolution of shijiuhu lake inferred from lake sediments. Quaternary Sciences, 2009, 29(2) : 248-255. [姚书春, 薛滨. 石臼湖近代环境演化历史. 第四纪研究, 2009, 29(2) : 248-255.]

[58] Cheng LJ, Xue B, Yao SC et al. Response of Cladocera fauna to environmental change based on sediments from Shengjin Lake, a Yangtze River-connected lake in China. Quaternary International, 2020, 536: 52-59. DOI: 10.1016/j. quaint. 2019.11.044.

[59] Cao YM, Zhang EL, Tang HQ et al. Combined effects of nutrients and trace metals on chironomid composition and morphology in a heavily polluted lake in central China since the early 20th century. Hydrobiologia, 2016, 779(1) : 147-159. DOI: $10.1007 /$ s10750-016-2810-y.

[60] Chen J, Jilili A, Ma L. Variation of the organic matter content of the sediment abstracted from Sayram Lake and its environmental significance. Journal of Glaciology and Geocryology, 2016, 38(3): 761-768. [ 陈京, 吉力力・阿不都外力, 马龙. 赛里木湖沉积物有机质变化特征及其环境信息. 冰川冻土, 2016, 38(3): 761-768.]

[61] Zhang YD, Yu JL, Su YL et al. Long-term changes of water quality in aquaculture-dominated lakes as revealed by sediment geochemical records in Lake Taibai (Eastern China). Chemosphere, 2019, 235: 297-291. DOI: 10.1016/j.chemosphere.2019.06.179.

[62] Ni ZK, Li YJ, Wang SR et al. The sources of organic carbon and nitrogen in sediment of Taihu Lake. Acta Ecologica Sini$c a, 2011,31(16)$ : 4661-4670. [ 倪兆奎, 李跃进, 王圣瑞等. 太湖沉积物有机碳与氮的来源. 生态学报, 2011, 31 (16) : 4661-4670.

[63] Ruan XC, Ye HP, Lu Q et al. Historical record, source, and toxicity assessment of sedimentary organic matter using molecular composition of hydrocarbons in an urban lake, Wuhan, China. Journal of Soils and Sediments, 2018, 18(5): 2081-2092. DOI: 10.1007/s11368-018-1956-9.

[64] Shen BB, Wu JL, Zhou JC et al. Tracking recent environmental changes in Lake Wanghu, China: A multivariate analysis of lipid biomarkers in sediments. Hydrobiologia , 2019, 829(1) : 281-290. DOI: 10.1007/s10750-018-3839-x.

[65] Gui ZF, Xue B, Yao SC et al. Catchment erosion and trophic status changes over the past century as recorded in sediments from Wudalianchi Lake, the northernmost volcanic lake in China. Quaternary International, 2012, 282 : 163-170. DOI: 10.1016/j.quaint.2012.05.012.

[66] Wu JL, Ma L, Yu H et al. Sediment geochemical records of environmental change in Lake Wuliangsu, Yellow River Basin, North China. Journal of Paleolimnology, 2013, 50(2) : 245-255. DOI: 10.1007/s10933-013-9718-6.

[67] Zhang EL, Liu EF, Jones R et al. A 150-year record of recent changes in human activity and eutrophication of Lake Wushan from the middle reach of the Yangze River, China. Journal of Limnology, 2010, 69(2) : 235. DOI: 10.4081/jlimnol. 2010.235 .

[68] Liu YY, Chen GJ, Hu K et al. Biological responses to recent eutrophication and hydrologic changes in Xingyun Lake, southwest China. Journal of Paleolimnology, 2017, 57(4) : 343-360. DOI: 10.1007/s10933-017-9952-4.

[69] Wu H, Chang FQ, Zhang HC et al. Changes of organic C and N stable isotope and their environmental implication during the past 100 years of Lake Yilong. Chinese Journal of Ecology, 2020, 39( 8) : 2478-2487. DOI: 10.13292/j.1000-4890. 202008.010. [ 吴汉, 常凤琴, 张虎才等. 异龙湖近百年来湖泊沉积物有机碳、氮稳定同位素变化及其环境指示意 义. 生态学杂志, $2020,39(8): 2478-2487$.

[70] Xiao HF, Zang SY, Meng ZH et al. Analysis on evolution of total nitrogen and organic matter in sediment of yamenxi lake of Lianhuan lake. Natural Science Journal of Harbin Normal University, 2010, 26(5) : 74-76, 91. [肖海丰, 藏淑英, 孟 昭虹等. 连环湖牙门喜泡沉积物总氮、有机质演化分析. 哈尔滨师范大学自然科学学报, 2010, 26 (5): 74$76,91$.

[71] Liu YL, Zhang EL, Liu EF et al. TOC and Black Carbon records in sediment of Lake Yangzong, Yunnan Province under the influence of human activities during the past century. J Lake Sci, 2017, 29(4) : 1018-1028. DOI: 10.18307/2017. 0426. [刘一兰, 张恩楼, 刘恩峰等. 人类活动影响下的云南阳宗海近百年有机碳与黑炭湖泊沉积记录. 湖泊科 学, 2017, 29(4): 1018-1028.]

[72] Rural Social and Economic Survey Department, National Bureau of Statistics ed. China County Statistical Yearbook-2018. Beijing: China Statistics Press, 2019. [国家统计局农村社会经济调查司. 中国县域统计年鉴—2018. 北京: 中国 统计出版社, 2019.] 
[73] Nanjing Institute of Geography \& Limnology Chinese Academy Science ed. Research report of Chinese lakes. Beijing: Science Press, 2019. [中国科学院南京地理与湖泊研究所. 中国湖泊调查报告. 北京:科学出版社, 2019.]

[74] Ning X, Qin Y, Cui Y et al. 10-year average hydrothermal condition change kilometer grid dataset of China( 1951-2010) [DB/OL]. Global Change Data Warehouse, 2016. DOI: 10.3974/geodb.2016.01.07.V1. [宁晓菊, 秦耀辰, 崔耀平等. 中国 10 年平均水热条件变化公里网格数据集 $(1951-2010)[\mathrm{DB} / \mathrm{OL}]$. 全球变化数据仓储, 2016.]

[75] Zeileis A, Leisch F, Hornik K et al. Strucchange: AnRPackage for testing for structural change in linear regression models. Journal of Statistical Software, 2002, 7(2) : 1-38. DOI: 10.18637/jss.v007.i02.

[76] Cluis DA. Visual techniques for the detection of water quality trends: Double-mass curves and CUSUM functions. Environmental Monitoring and Assessment, 1983, 3(2) : 173-184. DOI: 10.1007/BF00398846.

[77] Tilman D. Global environmental impacts of agricultural expansion: The need for sustainable and efficient practices. Proceedings of the National Academy of Sciences of the United States of America, 1999, 96(11) : 5995-6000. DOI: 10.1073/ pnas.96.11.5995.

[78 ] Gao XZ, Ma WQ, Du S et al. Current status and problems of fertilization in China. Chinese Journal of Soil Science, 2001, 32(6) : 258-261. [高祥照，马文奇，杜森等. 我国施肥中存在问题的分析. 土壤通报，2001，32(6): 258-261.]

[79] China Agricultural Yearbook Editorial Board ed. China Agricultural Yearbook 1981. Beijing: Agricultural Press, 1981: 55-64. [中国农业年鉴编辑委员会. 中国农业年鉴 1981. 北京: 农业出版社, 1981: 55-64.]

[ 80] Zhang WL, Wu SX, Ji HJ et al. Estimation of agricultural non-point source pollution in China and the alleviating strategies I . Estimation of agricultural non-point source pollution in China in early 21 century. Scientia Agricultura Sinica, 2004, 37 (7) : 1008-1017. [张维理, 武淑霞, 冀宏杰等. 中国农业面源污染形势估计及控制对策 I. 21 世纪初期中国农业 面源污染的形势估计. 中国农业科学, 2004, 37(7): 1008-1017.]

[81] Fu Q, Zhu YQ, Sun JL et al. Spatial patterns and gravity centers curve of livestock and poultry breeding in China. Acta Geographica Sinica, 2012, 67(10): 1383-1398. [付强, 诸云强, 孙九林等. 中国畜禽养殖的空间格局与重心曲线 特征分析. 地理学报, 2012, 67(10): 1383-1398.]

[82] Wu WF, Jin L, Yang NS. Trend in lake fishery development of China. J Lake Sci, 1991, 3(1) : 82-86. DOI: 10.18307/ 1991.0111. [吴万夫，金乐，杨宁生. 论中国湖泊渔业发展趋势. 湖泊科学, 1991, 3(1): 82-86.]

[83] Folke C, Carpenter S, Walker B et al. Regime shifts, resilience, and biodiversity in ecosystem management. Annual Review of Ecology, Evolution, and Systematics, 2004, 35(1) : 557-581. DOI: 10.1146/annurev.ecolsys.35.021103.105711.

[84] He CF, Hu XQ. Evolution of Chinese industrial geography since reform and opening-up. Acta Geographica Sinica, 2019, 74(10) : 1962-1979. DOI: 10.11821/dlxb201910002. [贺灿飞, 胡绪千. 1978 年改革开放以来中国工业地理格局演 变. 地理学报, 2019, 74(10): 1962-1979.]

[85] Bennett EM, Carpenter SR, Caraco NF. Human impact on erodable phosphorus and eutrophication: A global perspective. BioScience, 2001, 51(3) : 227. DOI: 10.1641/0006-3568(2001)051[0227: hioepa]2.0.co;2.

[86] Qin BQ. Approaches to mechanisms and control of eutrophication of shallow lakes in the middle and lower reaches of the yangze river. J Lake Sci, 2002, 14(3) : 193-202. DOI : 10.18307/2002.0301. [秦伯强. 长江中下游浅水湖泊富营养 化发生机制与控制途径初探. 湖泊科学, 2002, 14(3): 193-202.]

[87] Zhang H, Tao SL, Tang ZY et al. Lake area changes in jing-Jin-ji region in recent 30 years. Acta Scientiarum Naturalium Universitatis Pekinensis, 2020, 56(2) : 324-330. DOI: 10.13209/j.0479-8023.2019.123. [张恒, 陶胜利, 唐志尧等. 近 30 年京津冀地区湖泊面积的变化. 北京大学学报: 自然科学版, 2020, 56(2) : 324-330.]

[ 88 ] Gui ZF, Xue B, Yao SC et al. Responses of lakes in the Songnen Plain to climate change. J Lake Sci, 2010, 22 (6) : 852 861. DOI: 10.18307/2010.0607. [桂智凡, 薛滨, 姚书春等. 东北松嫩平原区湖泊对气候变化响应的初步研究. 湖 泊科学, $2010,22(6): 852-861$.]

[ 89] Tao S, Fang J, Zhao X et al. Rapid loss of lakes on the Mongolian Plateau. Proceedings of the National Academy of Sciences of the United Statses of America, 2015, 112(7) : 2281-2286. DOI: 10.1073/pnas.1411748112. 

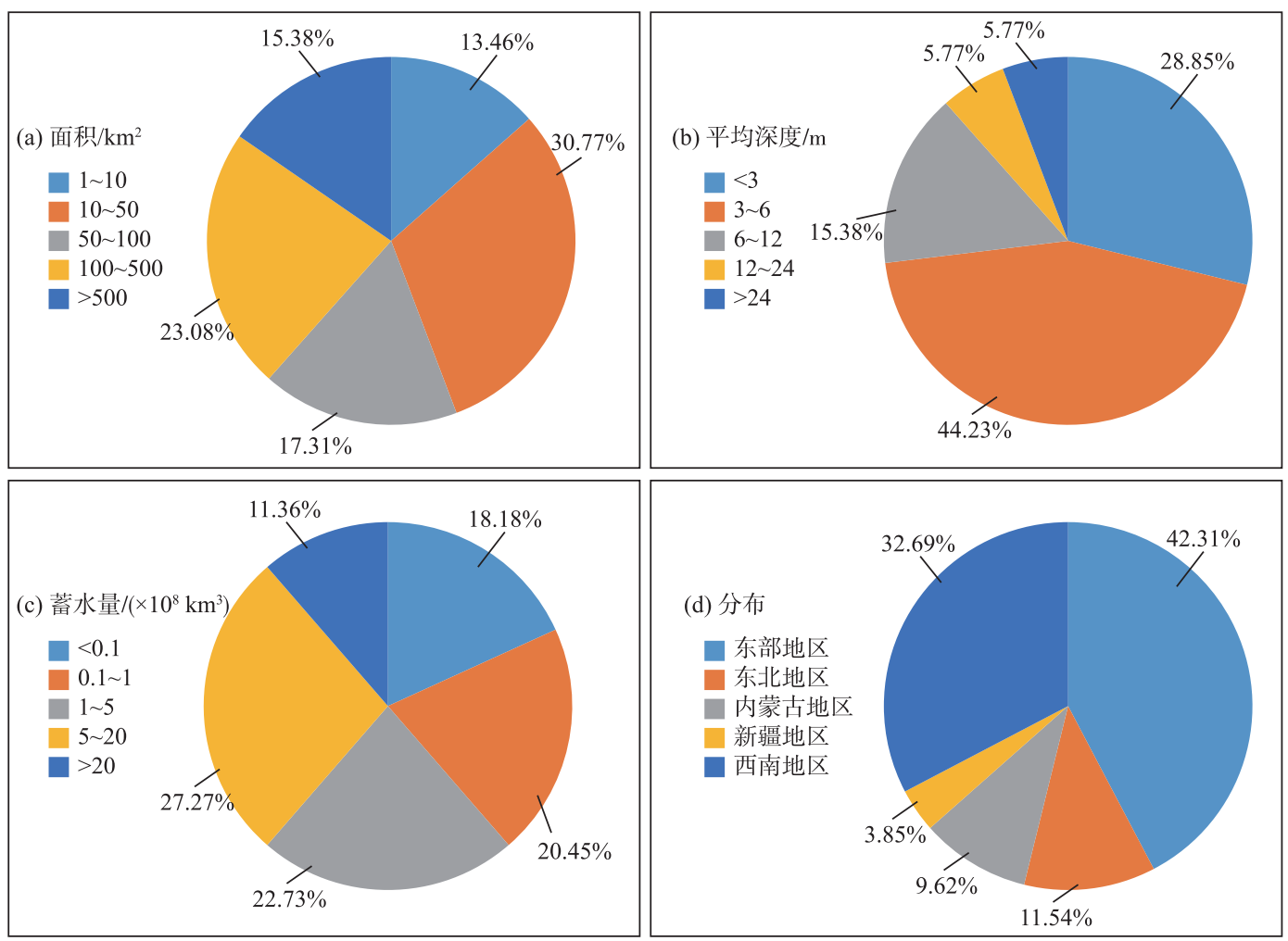

附图 I 湖泊物理属性和分布

Attached Fig. I Physical properties and distribution of lakes 
附表 I 研究涉及湖泊位置 *

Attached Tab. I Location of lakes involved in the study

\begin{tabular}{|c|c|c|c|c|c|c|c|}
\hline 湖泊 & 经度 $/\left({ }^{\circ}\right)$ & 纬度 $/\left(^{\circ}\right)$ & 城乡分异 & 湖泊 & 经度 $/\left({ }^{\circ}\right)$ & 纬度 $/\left(^{\circ}\right)$ & 城乡分异 \\
\hline 艾比湖 & 88.96 & 44.88 & 中间湖泊 & 克钦泡 & 124.30 & 47.30 & 中间湖泊 \\
\hline 博斯腾湖 & 87.04 & 41.97 & 中间湖泊 & 龙感湖 & 116.49 & 30.07 & 中间湖泊 \\
\hline 白洋淀 & 115.95 & 38.92 & 中间湖泊 & 沾沽湖 & 100.78 & 27.71 & 乡村湖泊 \\
\hline 巢湖 & 117.53 & 31.57 & 城郊湖泊 & 龙江泡 & 123.15 & 46.86 & 乡村湖泊 \\
\hline 长荡湖 & 119.55 & 31.62 & 中间湖泊 & 梁子湖 & 114.51 & 30.23 & 中间湖泊 \\
\hline 程海 & 100.65 & 26.55 & 乡村湖泊 & 南四湖 & 116.96 & 34.87 & 城郊湖泊 \\
\hline 陈普海子 & 106.82 & 40.65 & 乡村湖泊 & 南渏湖 & 118.96 & 31.11 & 乡村湖泊 \\
\hline 长桥海 & 103.34 & 23.45 & 中间湖泊 & 杞麓湖 & 102.72 & 24.15 & 中间湖泊 \\
\hline 长寿湖 & 107.30 & 29.92 & 乡村湖泊 & 清水海 & 103.11 & 25.60 & 乡村湖泊 \\
\hline 柴窝铺 & 87.90 & 43.50 & 乡村湖泊 & 三岔湖 & 104.27 & 30.30 & 乡村湖泊 \\
\hline 滇池 & 102.69 & 24.91 & 城郊湖泊 & 石臼湖 & 118.88 & 31.47 & 中间湖泊 \\
\hline 达里湖 & 116.64 & 43.29 & 乡村湖泊 & 升金湖 & 117.07 & 30.38 & 中间湖泊 \\
\hline 大理西湖 & 100.05 & 26.01 & 乡村湖泊 & 三里七湖 & 114.95 & 30.11 & 城郊湖泊 \\
\hline 淀山湖 & 120.96 & 31.12 & 城郊湖泊 & 赛里木湖 & 81.17 & 44.60 & 乡村湖泊 \\
\hline 大屯海 & 103.30 & 23.41 & 中间湖泊 & 太白湖 & 115.81 & 29.97 & 乡村湖泊 \\
\hline 洞庭湖 & 112.96 & 29.33 & 乡村湖泊 & 太湖 & 120.19 & 31.20 & 城郊湖泊 \\
\hline 洱海 & 100.19 & 25.82 & 中间湖泊 & 武昌东湖 & 114.40 & 30.56 & 城郊湖泊 \\
\hline 抚仙湖 & 102.89 & 24.58 & 中间湖泊 & 网湖 & 115.33 & 29.87 & 中间湖泊 \\
\hline 固城湖 & 118.90 & 31.30 & 中间湖泊 & 三池 & 126.22 & 48.73 & 中间湖泊 \\
\hline 湖北洪湖 & 113.34 & 29.86 & 城郊湖泊 & 五池 & 126.14 & 48.78 & 中间湖泊 \\
\hline 红枫湖 & 106.41 & 26.50 & 中间湖泊 & 乌梁素海 & 108.85 & 40.96 & 乡村湖泊 \\
\hline 黄盖湖 & 113.55 & 29.70 & 乡村湖泊 & 武山湖 & 115.59 & 29.91 & 城郊湖泊 \\
\hline 红碱淖 & 108.89 & 39.09 & 乡村湖泊 & 星云湖 & 102.80 & 24.37 & 中间湖泊 \\
\hline 呼伦湖 & 117.40 & 48.94 & 乡村湖泊 & 异龙湖 & 102.60 & 23.67 & 中间湖泊 \\
\hline 海西海 & 99.96 & 26.48 & 乡村湖泊 & 牙门喜泡 & 124.09 & 46.76 & 乡村湖泊 \\
\hline 鸿雁泡 & 123.51 & 47.34 & 中间湖泊 & 阳宗海 & 103.00 & 24.91 & 乡村湖泊 \\
\hline 剑湖 & 99.93 & 26.49 & 乡村湖泊 & 涨渡湖 & 114.70 & 30.65 & 中间湖泊 \\
\hline 江西洪湖 & 116.91 & 28.17 & 中间湖泊 & & & & \\
\hline
\end{tabular}

* 城乡分异结合湖岸、湖泊流域和所在盆地是否存在城镇和城镇规模综合判断. 